\title{
Pourquoi nous devrions penser aux Italiens
}

\author{
Bettina Maeschlia, Erminia Gagliotta ${ }^{a}$, Philip Bruggmann ${ }^{a, b}$ \\ a Hépatite Suisse, Zurich; ${ }^{\text {b }}$ Arud Zentrum für Suchtmedizin, Zurich
}

\begin{abstract}
Les Italiennes et Italiens de Suisse âgés de plus de 60 ans sont particulièrement touchés par l'hépatite C. La plupart ont été contaminés dans les années 1950 et 1960 dans les milieux paramédicaux de leur pays d'origine. L'hépatite $C$ chronique pouvant désormais être guérie sans complications, il est intéressant de tester ce groupe de population.
\end{abstract}

L.F. a quitté la Sicile pour s'installer en Suisse alors qu'il était un jeune homme. L'hépatite $C$ lui a été diagnostiquée lors d'un examen médical de routine en 2006. Le diagnostic lui parvient de manière totalement inattendue, car il n'a aucune douleur. En plus des contrôles réguliers (échographie abdominale, fibroscan et analyse de sang), les médecins lui proposent une thérapie à l'interféron, qu'il refuse en raison des lourds effets secondaires et de l'évolution asymptomatique de sa maladie. Ce n'est qu'en 2020 que le patient suit une thérapie. Cette fois-ci avec les médicaments antiviraux approuvés depuis quelques années maintenant. Le traitement prévoit la prise d'un seul comprimé par jour, dure au total 84 jours et ne présente pratiquement aucun symptôme concomitant. L.F. est déjà sain pendant la thérapie et le reste à ce jour. Grâce à la guérison de l'hépatite $\mathrm{C}$, le risque de maladies secondaires a pu être éliminé à son grand soulagement.

\section{Italiens quatre fois plus susceptibles d'être affectés}

L'histoire de cet Italien du Sud, aujourd'hui âgé de 76 ans, n'est pas atypique. Il y a environ 32000 personnes vivant avec l'hépatite $C$ en Suisse. Selon une évaluation des données de la Cohorte suisse de l'hépatite $\mathrm{C}$ et du système de déclaration obligatoire de l'Office fédéral de la santé publique (OFSP), les personnes de plus de 60 ans nées en Italie et vivant en Suisse ont presque quatre fois plus de risques d'être touchées par l'hépatite C que le reste de la population suisse [1]. Ces chiffres s'expliquent par les cures de vitamines et autres pratiques paramédicales opérées dans le Sud de l'Italie dans les années 1950 et 1960. A cette époque, un personnel mal formé traitait toute la population des villages italiens par des injections. Il n'était pas rare d'utiliser la même seringue en verre, insuffisamment stérilisée [2-4].

\section{L'hépatite C est aujourd'hui curable}

Ces dernières années ont révolutionné le traitement de l'hépatite C. Pendant longtemps, les thérapies à base d'interféron associées à la ribavirine étaient la seule option thérapeutique pour l'hépatite $C$ chronique. Comme ces thérapies étaient longues (jusqu'à un an ou plus) et qu'elles avaient parfois des effets secondaires très graves, seuls les patients et patientes présentant des lésions hépatiques avancées étaient traités.

En 2014, les premiers agents antiviraux à action directe (AAD) sont arrivés sur le marché. Au début, ils devaient encore être administrés en même temps que l'interféron. Aujourd'hui, les thérapies combinées qui ne nécessitent pas d'interféron sont la norme. Un ou trois comprimés par jour sont encore pris pendant 8 ou 12 semaines. Le taux de guérison est de plus de $96 \%$.

Avec les nouvelles thérapies, les recommandations de traitement ont également changé: Aujourd'hui, toutes les personnes infectées de façon chronique par l'hépatite $C$ devraient se voir proposer un traitement [5]. L'infection par l'hépatite $C$ progresse généralement lentement sur des années et des décennies, conduisant à une cirrhose du foie dans 5 à $20 \%$ des cas. Chaque année, 2 à $4 \%$ des patients atteints de cirrhose développent un carcinome hépatocellulaire (CHC). L’hépatite $\mathrm{C}$ est encore aujourd'hui l'une des causes les plus fréquentes de $\mathrm{CHC}$, ainsi qu'un motif fréquent de transplantation hépatique. D'une part, le cancer du foie 


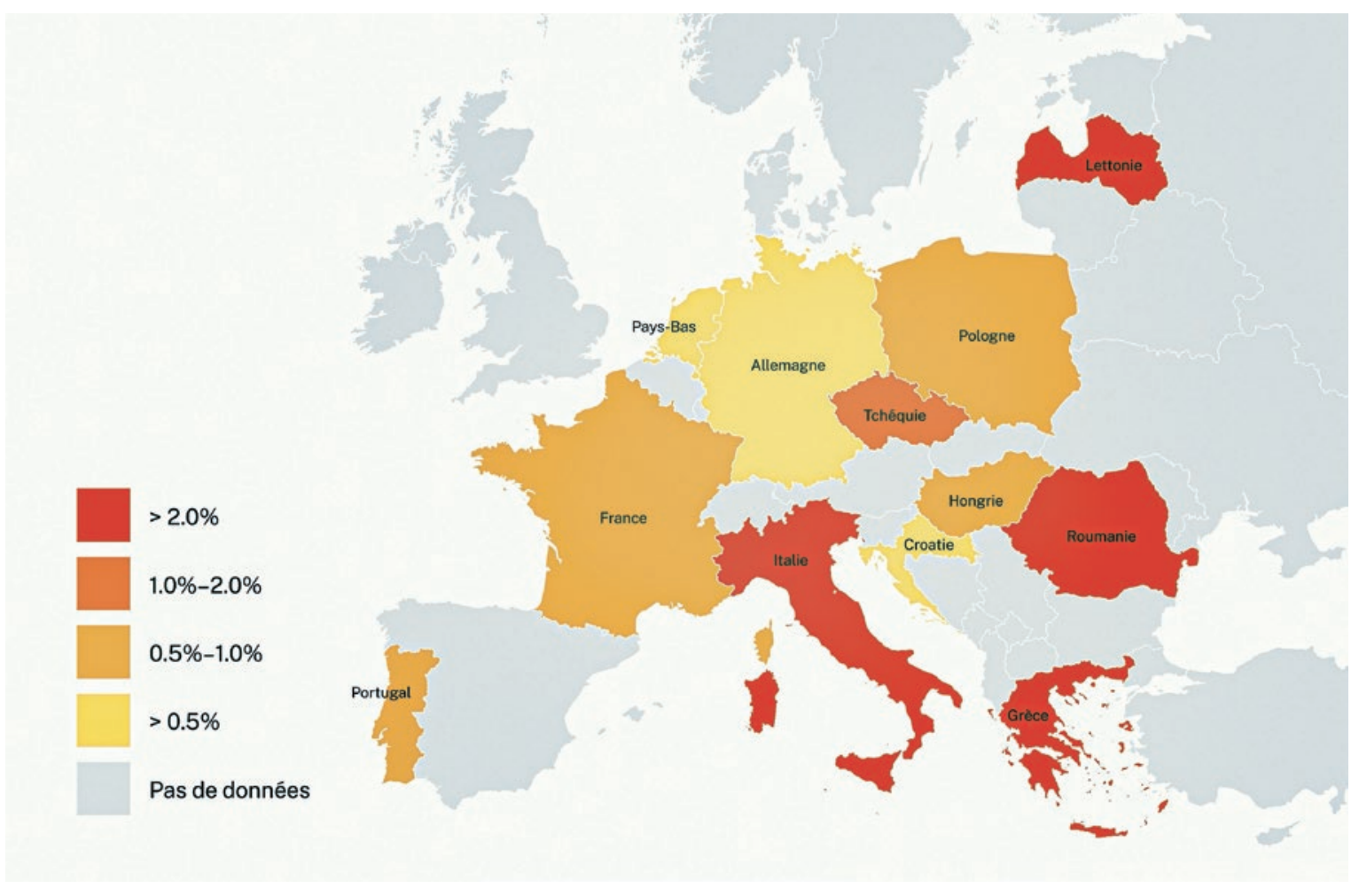

Prévalence de l'hépatite $\mathrm{C}$ en Europe (adaptée de: ECDC Technical Report: Prevention of hepatitis B and C in the EU/EEA and the UK, novembre 2020 - copyright Hépatite Suisse).

et la transplantation peuvent être évités grâce à de nouvelles options de traitement très efficaces. D’autre part, l'hépatite $\mathrm{C}$ est également à l'origine de diverses autres maladies en dehors du foie, qui peuvent être prévenues par un traitement approprié: soit le diabète, les maladies cardiovasculaires et les lymphomes. En outre, une grande partie des patients et patientes se plaignent d'une fatigue importante qui, dans certains cas, peut également entraîner une incapacité de travail [6].

\section{Traitement par la médecine familiale}

Du point de vue de la santé publique, il convient de diagnostiquer et de traiter l'hépatite $C$ chronique qui, comme pour L.F., est souvent asymptomatique ou ne présente aucun symptôme spécifique. Grâce aux thérapies et à un petit nombre de nouvelles infections, il serait possible aujourd'hui d'éliminer l'hépatite $C$.

Aujourd'hui, les AAD ne peuvent être prescrits que par des spécialistes (infectiologues, gastro-entérologues ou médecins spécialistes de la toxicomanie ayant une expérience du traitement de l'hépatite C). Cependant, les thérapies étant simples, les médecins généralistes peuvent traiter eux-mêmes leur patientèle via le projet HepCare (www.hepcare.ch).
Comme le montre l'exemple de L.F., nous devons lors des tests penser aux Italiennes et Italiens plus âgés qui sont arrivés chez nous dans les années 1950 et 1960. Des enquêtes menées dans les années 1990 ont révélé des taux de prévalence allant jusqu'à $12 \%$ pour les anticorps de l'hépatite $\mathrm{C}$ dans certains villages du sud de l'Italie [2]. Le pays supporte encore ce fardeau aujourd'hui: le dernier rapport du Centre européen de contrôle des maladies estime la prévalence de l'hépatite $C$ dans la population générale en Italie à 3,9\% [7], soit l'une des plus élevées d'Europe (voir la figure «Prévalence de l'hépatite $C$ en Europe»).

\section{Test d'anticorps lors du bilan de santé}

Outre les Italiennes et Italiens, les immigrants d'Espagne et du Portugal et des pays des Balkans sont également touchés de manière disproportionnée par l'hépatite C [1]. Ces personnes ont été infectées dans de nombreux cas dans le domaine médical. Le principal risque de transmission dans le reste de la population est la consommation de drogues par voie intraveineuse. Cela inclut également les personnes qui ont brièvement expérimenté la drogue dans le passé et qui ont ainsi été infectées. Les autres risques sont les transfusions sanguines et les procédures médicales (den- 
taires) effectuées avant les années 1990. Des tatouages ou piercings réalisés dans des conditions non hygiéniques peuvent également entraîner une infection. Dans la population générale, on observe une nette répartition par âge des infections avérées: la cohorte de naissance 1950-1985 représente les trois quarts des personnes touchées [8].

Il est donc intéressant, par exemple dans le cadre de bilans de santé, de proposer un test de détection des anticorps anti-VHC pour la cohorte de naissance 1950-1985 ou pour les personnes consommant actuellement ou ayant consommé des drogues. Idéalement, lors de la demande d'analyses pour la recherche d'anticorps, la détermination de l'ARN du VHC devrait être demandée en même temps en cas de résultat positif.

Nous devrions penser aux Italiennes et Italiens plus âgés quand il s'agit de l'hépatite $\mathrm{C}$. Compte tenu de la facilité du diagnostic, du traitement élémentaire et des fortes chances de réussite, il est logique que les Italiennes et Italiens de première génération ayant émigré, ainsi que les populations susmentionnées, soient testés pour l'hépatite $C$ au moins une fois dans leur vie afin de pouvoir être traités en cas de résultat positif.

\section{La Stratégie Hépatite Suisse}

L'association Hépatite Suisse coordonne la Stratégie suisse contre l'hépatite, un réseau de plus de 80 experts bénévoles dans toute la Suisse qui vise à éliminer les hépatites $B$ et $C$ d'ici 2030. Cet objectif a également été fixé par l'Organisation mondiale de la Santé (OMS) au niveau global. L'élimination est réaliste: une vaccination efficace protège contre l'infection par I'hépatite $B$. Aujourd'hui, I'hépatite $C$ peut presque toujours être guérie grâce à des thérapies efficaces. Cependant, trop de personnes atteintes ne sont pas diagnostiquées ni traitées. Pour atteindre les objectifs d'élimination, davantage de personnes doivent être diagnostiquées et traitées.

Plus d'informations: www.hepatitis-schweiz.ch; www.hepcare.ch (les prestataires de soins primaires traitent I'hépatite C).

\section{Références}

1 Bertisch B, Giudici F, Negro F, Moradpour D, Müllhaupt B, Moriggia $\mathrm{A}$, et al. Characteristics of Foreign-Born Persons in the Swiss Hepatitis C Cohort Study: Implications for Screening Recommendations. PLOS ONE 11(5): e0155464.

2 Guadagnino V, Stroffolini T, Rapicetta M, Costantino A, Kondili LA Menniti-Ippolito F, et al. Prevalence, risk factors, and genotype distribution of hepatitis $C$ virus infection in the general population: a community-based survey in southern Italy. Hepatology. 1997;26:1006-11.

3 Andriulli A, Stroffolini T, Mariano A, Valvano MR, Grattagliano, Ippolito AM, et al. Declining prevalence and increasing awareness of hepatitis $C$ virus infection in Italy: a population-based survey. Eur J Intern Med. 2018;53:79-84.

4 Kondili LA, Gamkrelidze I, Blach S, Marcellusi A, Galli M, Petta S, et al. Optimization of hepatitis $C$ virus screening strategies by birth cohort in Italy. Liver Int. 2020;40:1545-55.

5 Swiss Association for the Study of the Liver SASL, Société Suisse de Gastro-entérologie SSG et Société Suisse d'Infectiologie SGINF. Update Expert Opinion Statement: Treatment of Chronic Hepatitis C, Januar 2021.

6 Sarkar S, Jiang Z, Evon DM, Wahed AS, Hoofnagle JH. Fatigue before, during and after antiviral therapy of chronic hepatitis C: results from the Virahep-C study. J Hepatol. 2012;57(5):946-52.

7 ECDC Technical Report: Prevention of hepatitis B and C in the EU/ EEA and the UK, November 2020.

8 Bruggmann P, Richard J-L, on behalf of the Swiss Hepatitis C Cohort Study Group. Birth year distribution in reported hepatitis $C$ cases in Switzerland. European Journal of Public Health. 2015 (Feb); 25(1):141-3.

\section{L'essentiel en bref}

- Les personnes de plus de 60 ans nées en Italie et vivant en Suisse ont presque quatre fois plus de risques d'être touchées par l'hépatite $\mathrm{C}$ que le reste de la population suisse.

- Toutes les personnes infectées de façon chronique par I'hépatite $\mathrm{C}$ devraient se voir proposer un traitement.

- Aujourd'hui, les thérapies combinées qui ne nécessitent pas d'interféron sont la norme.

- Les médecins généralistes peuvent traiter eux-mêmes leur patientèle via le projet HepCare (www.hepcare.ch). 